\title{
Conhecimento materno no período gestacional sobre saúde bucal: um estudo piloto
}

\section{Mother knowledge about oral health in pregnancy: a pilot study}

\author{
Mateus Machado Delfinoํㅡ, Elizangela Partata Zuza ${ }^{2}$ Juliana Rico Pires², Maria Cristina Meinberg², \\ Alex Tadeu Martins², Fabiano de Sant'Ana dos Santos², Fábio Luiz Ferreira Scannavino² \\ ${ }^{1}$ Mestrando em Odontologia, Faculdade de Odontologia de Araraquara, UNESP / FOAr - Araraquara (SP) - \\ Brasil. \\ ${ }^{2}$ Professores do curso de Odontologia do Centro Universitário da Fundação Educacional de Barretos, \\ UNIFEB - Barretos (SP) - Brasil. \\ ${ }^{3}$ Cirurgiã-dentista e servidora da secretaria municipal de saúde - Barretos (SP) - Brasil.
}

\begin{abstract}
Resumo
Objetivo: Analisar o conhecimento de gestantes sobre a saúde bucal e os procedimentos preventivos realizados pela mãe após o nascimento do bebê. Métodos: Este estudo é do tipo epidemiológico transversal, através de amostragem por conveniência. Participaram gestantes multíparas e primíparas, da rede municipal de saúde e de convênio de saúde particular, no período de março a agosto do ano de 2015. Foram incluídas, no estudo, 89 gestantes com idade entre 15 e 40 anos. Aplicou-se um questionário contendo perguntas distintas direcionadas para mães pela primeira vez e multíparas, abrangendo questões sobre as condições sociais e outras variáveis, como acompanhamento do pré-natal. As respostas foram analisadas por meio do software SPSS versão 15/2012 e, posteriormente, foram procedidas as análises estatísticas descritiva e inferencial, por meio do teste qui-quadrado $\chi^{2}$. Resultados: O presente estudo mostrou diferença estatisticamente significante entre as gestantes grávidas pela primeira vez com idade inferior aos 18 anos, solteiras e com nível de escolaridade superior. Conclusão: As gestantes demonstraram conhecimento sobre a saúde bucal na gravidez e a importância da realização do pré-natal odontológico.
\end{abstract}

Palavras-chave: serviços de saúde materno-infantil; serviços preventivos de saúde; educação em saúde bucal.

\begin{abstract}
Objective: To analyze the knowledge of pregnant women about oral health and the preventive procedures performed by the mother after the baby is born. Methods: This is a cross-sectional epidemiological study using convenience sampling. Participants were multiparous and primiparous pregnant women, recruited from the municipal health network and private health insurance, from March to August 2015. The study included 89 pregnant women aged 15-40 years. We applied a questionnaire with different questions addressed to primiparous and multiparous mothers. Questionnaires included questions about social conditions, and other variables such as prenatal follow-up. The responses were analyzed using the SPSS software version $15 / 2012$ and later, the descriptive and inferential statistical analyzes were carried out using the chi-square test $\chi^{2}$. Results: The present study showed a statistically significant difference between pregnant women for the first time under the age of eighteen, single and with a higher education level. Conclusion: Pregnant women demonstrated knowledge about oral health in pregnancy and the importance of performing prenatal dentistry.
\end{abstract}

Keywords: maternal-child health services; preventive health services; health education, dental.

Autor correspondente: Fábio Luiz Ferreira Scannavino - Av. Prof Roberto Frade Monte, 386 CEP:14783-226 - Barretos (SP), Brasil - E-mail: flscannavino@gmail.com

Recebido em: 25/10/2017

Aceito em: 23/07/2018

https://doi.org/10.4322/1980-0029.172017 


\section{Introdução}

A gestação constitui-se num fenômeno fisiológico que, na maior parte dos casos, tem sua evolução sem intercorrências. As alterações durante a gravidez podem ser explicadas pelo desequilíbrio da atividade metabólica ocasionada pela elevação e liberação de taxas hormonais, inclusive a progesterona (MEDEIROS et al., 2000). Essas alterações repercutem na fisiologia bucal, modificando o equilíbrio normal da boca, podendo exacerbar o processo carioso e infecções periodontais. Ressalta-se que o período gestacional não é o responsável direto por estas alterações, mas pode agravar a inflamação gengival preexistente e lesões de cárie, principalmente se houver negligência da higiene bucal (VERGNES et al., 2012).

No período gestacional, a futura mãe preocupa-se e tem interesse não somente pela própria saúde, mas também com a do feto. Desta forma, gera-se um maior interesse da mãe na busca de informações que contribuirão para a sua saúde e, sobretudo, a do bebê, que está em desenvolvimento. Esse momento é extremamente valioso para a abordagem de ações educativas e preventivas com gestantes em relação aos cuidados com a saúde bucal e à introdução de bons hábitos desde o início da vida da criança (FINKLER et al., 2004; CODATO et al., 2011).

Após o nascimento do bebê e em decorrência do rápido crescimento durante o primeiro ano de vida, a infância torna-se um dos períodos mais críticos do ciclo vital. Dessa forma, a nutrição e, mais especificamente, a aleitamento natural, são essenciais para o desenvolvimento humano. $\mathrm{O}$ aleitamento materno oferece ao bebê um adequado desenvolvimento ósseo e muscular, garantindo, assim, a inter-relação entre o sistema estomatognático e os demais órgãos e funções (BATISTA et al., 2011).

A cárie dentária é a doença mais crônica na infância, consistindo em um problema de saúde pública. De acordo com a Academia Americana de Odontopediatria, o fator de risco para o desenvolvimento da doença cárie é o aleitamento com livre demanda após a erupção dos dentes e o uso de chupetas imersas em substâncias açucaradas. Outro fator de grande importância em relação à cárie precoce na infância é a mamadeira no intervalo de sono e antes de dormir, sem a higienização da boca, posteriormente (AMERICAN ACADEMY OF PEDIATRIC DENTISTRY, 2015).
Os hábitos bucais se instalam com maior frequência em crianças que não tiveram amamentação natural (MOIMAZ et al., 2011). O aleitamento no peito materno, além de alimentar o bebê, tem a função de satisfazer a sucção devido à ação muscular exercida durante a sucção. Quando a criança é amamentada por mamadeiras, o fluxo de leite é bem maior que a amamentação natural, portanto a criança se satisfaz em menor tempo e mínimo de esforço. O prazer emocional com relação ao impulso da sucção não é atingido e a criança procura, por isso, algo substituto, como dedo, chupeta e objetos, para satisfazer-se (MOIMAZ et al., 2011).

Face à relevância da prevenção de doenças bucais e, por conseguinte, sistêmicas, na gravidez, torna-se imprescindível a instalação de medidas educativo-preventivas frequentes às gestantes, bem como uma maior integração entre as classes médica e odontológica, visando a um melhor esclarecimento sobre a seguridade do tratamento odontológico (LANDERDAHL et al., 2007; BASTIANI et al., 2010). Assim, o objetivo deste trabalho foi analisar o conhecimento de gestantes sobre a saúde bucal e os aspectos preventivos envolvidos após o nascimento do bebê.

\section{Material e métodos}

Este estudo é do tipo epidemiológico transversal. As participantes foram gestantes multíparas e primíparas, cadastradas regularmente no pré-natal oferecido pela rede municipal de saúde e também por um convênio de saúde particular, na cidade de Barretos-SP, no período de março a agosto do ano de 2015. Foram incluídas, no estudo, 89 gestantes, de forma consecutiva, com idade entre 15 e 40 anos.

Um questionário especialmente elaborado pelos pesquisadores foi aplicado neste estudo piloto, contendo um total de 11 questões de múltipla escolha, sendo preenchido de forma voluntária, com a identidade da entrevistada mantida em sigilo. As gestantes eram convidadas, pessoalmente, a participarem de forma voluntária do estudo nas unidades básicas de saúde ou nos convênios particulares, sendo que o questionário era respondido em local reservado.

Um único pesquisador, treinado, realizou a aplicação do instrumento de coleta de dados impresso, pessoalmente, para que possíveis dúvidas pudessem ser prontamente esclarecidas 
às gestantes. Os questionários eram lacrados em envelopes individuais para posterior análise.

As participantes assinaram o Termo de Consentimento Livre e Esclarecido (TCLE). Este trabalho foi submetido ao Comitê de Ética em Pesquisa do Curso de Odontologia do Centro Universitário da Fundação Educacional de Barretos, sob o protocolo n. ${ }^{\circ} 30813414.1 .0000 .5433$.

Os questionários possuíam perguntas distintas, direcionadas para mães pela primeira vez primíparas e multíparas com mais de um filho, tendo em vista a experiência da maternidade ainda não vivida - primíparas - ou já vivenciada - multíparas, e outras variáveis, como acompanhamento do pré-natal, ingestão de complexos vitamínicos, aleitamento materno, conhecimento sobre hábitos de sucção não nutritivos e higiene bucal da mãe e do futuro bebê.

As respostas foram analisadas por meio do software SPSS versão 15/2012 e, posteriormente, procedidas as análises estatísticas descritiva e inferencial, por meio do Teste Qui-quadrado $\chi^{2}$.

\section{Resultados}

O presente estudo evidenciou, em termos de perfil das entrevistadas, a idade, o estado civil e a escolaridade. Não houve recusa em participar do estudo e, dessa forma, todas as 89 gestantes responderam de forma voluntária à pesquisa. Os resultados mostraram diferença estatisticamente significante entre as gestantes grávidas pela primeira vez com idade inferior aos 18 anos, solteiras e com nível de escolaridade superior, conforme a Tabela 1.

Em relação à realização do pré-natal das gestantes primíparas e multíparas, ambas o realizaram preferencialmente a partir do primeiro trimestre de gravidez (Tabela 2 ).

Sobre a amamentação, $52,7 \%$ das entrevistadas primíparas receberam orientação sobre a amamentação pelo médico e $23,6 \%$ destas, durante o pré-natal. Outrossim, 78,2\% das mães relataram pretender amamentar no peito pelo menos quatro vezes ou mais por dia e $45,5 \%$ delas, por até seis meses.

Em relação à amamentação, com as gestantes multíparas, a maioria $(52,7 \%)$ relatou que parte dos filhos advindos em gestações anteriores foi amamentada no peito exclusivamente até os seis meses de vida pós-natal (52,9\%), o que, segundo elas, garantiria ganho de peso $(50 \%)$ e preveniria doenças $(35,3 \%)$. Entretanto, as gestantes ressaltaram também que utilizaram a amamentação artificial $(85,3 \%)$, adquirindo a mamadeira $(79,4 \%)$ e oferecendo-a ao recém-nascido após o primeiro mês $(38,2 \%)$.

Quanto aos cuidados com a saúde bucal, tanto as primíparas quanto as multíparas realizaram tratamento odontológico durante a gravidez, buscando-o através do atendimento particular, conforme ilustra a Tabela 3.

Tabela 1. Análise comparativa do perfil sociodemográfico das participantes do estudo em Barretos-SP, Brasil, 2015

\begin{tabular}{|c|c|c|c|c|c|c|c|c|c|c|}
\hline \multirow{3}{*}{ Grupos } & \multicolumn{10}{|c|}{ Perfil dos Participantes } \\
\hline & \multicolumn{2}{|c|}{ Idade (anos) } & \multicolumn{4}{|c|}{ Estado Civil } & \multicolumn{4}{|c|}{ Escolaridade } \\
\hline & $<18$ & $>18$ & Solteira & Casada & Amasiada & Divorciada & $\begin{array}{c}\text { Até } \\
\text { Fundamental } \\
\end{array}$ & $\begin{array}{c}\text { Até } \\
\text { Médio }\end{array}$ & Superior & Técnico \\
\hline Primípara & $17^{*}$ & 38 & $26^{*}$ & 21 & 7 & 1 & 12 & 32 & $11 *$ & 0 \\
\hline Multípara & 4 & 30 & 8 & 18 & 8 & 0 & 9 & 20 & 1 & $4 *$ \\
\hline Valor de P & 0,0388 & $>0,05$ & 0,0472 & $>0,05$ & $>0,05$ & $>0,05$ & $>0,05$ & $>0,05$ & 0,0381 & 0,0092 \\
\hline
\end{tabular}

*Teste de Qui-quadrado $\chi^{2} ; \mathrm{P}<0,05$ indica diferença estatisticamente significante entre gestantes primíparas e multíparas.

Tabela 2. Análise sobre realização do Pré-natal em gestantes na cidade de Barretos-SP, Brasil, 2015

\begin{tabular}{|c|c|c|c|c|}
\hline \multirow{3}{*}{ Grupos } & \multicolumn{4}{|c|}{ Sobre Pré-Natal } \\
\hline & \multicolumn{2}{|c|}{ Faz Pré-natal } & \multicolumn{2}{|c|}{ Trimestre } \\
\hline & Sim & Não & $\begin{array}{c}\text { Desde o } 1^{\circ} \\
\text { trimestre }\end{array}$ & $\begin{array}{l}\text { A partir do } 2^{\circ} \\
\text { trimestre }\end{array}$ \\
\hline Primípara & 51 & 4 & 45 & 6 \\
\hline Multípara & 34 & 0 & 29 & 5 \\
\hline Valor de $\mathrm{P}$ & \multicolumn{2}{|c|}{0,1076} & \multicolumn{2}{|c|}{0,6923} \\
\hline
\end{tabular}

*Teste de Qui-quadrado $\chi^{2} ; \mathrm{P}>0,05$ não indica diferença estatisticamente significante entre gestantes primíparas e multíparas. 
Tabela 3. Análise sobre Saúde Bucal em gestantes primíparas e multíparas na cidade de Barretos-SP, Brasil, 2015

\begin{tabular}{|c|c|c|c|c|}
\hline \multirow[t]{2}{*}{ Grupos } & \multicolumn{2}{|c|}{$\begin{array}{c}\text { Realização do tratamento odontológico } \\
\text { é possível na gravidez? }\end{array}$} & \multicolumn{2}{|c|}{$\begin{array}{l}\text { Se sim, em qual modalidade } \\
\text { assistencial? }\end{array}$} \\
\hline & Sim & Não & Particular & Público \\
\hline Primípara & 52 & 3 & $14 *$ & 6 \\
\hline Multípara & 31 & 3 & 1 & $13 *$ \\
\hline Valor de P & \multicolumn{2}{|c|}{0,5380} & \multicolumn{2}{|c|}{0,0003} \\
\hline
\end{tabular}

*Teste de Qui-quadrado $\chi^{2} ; \mathrm{P}<0,05$ indica diferença estatisticamente significante entre gestantes primíparas e multíparas.

\section{Discussão}

A atenção odontológica dispensada às gestantes reveste-se de importância, considerando os avanços científicos e tecnológicos, em termos de diagnóstico e tratamento mais seguros, garantindo a integridade física e emocional de mãe e filho. No presente estudo, as gestantes primíparas e multíparas tinham idade superior a 18 anos, sendo que as primíparas, em sua maioria, eram solteiras, diferentemente das multíparas, cujo estado civil predominante era casada. $\mathrm{O}$ ensino médio foi o grau de escolaridade preponderante, diferindo dos resultados encontrados na literatura, provavelmente pela ausência de oportunidades e condições socioeconômicas desfavoráveis, caraterísticas presentes em países em desenvolvimento. (HASHIM, 2012; BAMANIKAR; KEE, 2013; GEORGE et al., 2013; CATÃO et al., 2015).

No estudo realizado, a adesão ao pré-natal pelas gestantes primíparas e multíparas foi considerado exitoso, provavelmente em função do reconhecimento, por parte das gestantes, da importância do programa preventivo, assim como a maciça divulgação dos pré-natais pelos profissionais da saúde, nas unidades básicas e nos postos de saúde, através de campanhas e programas educativos. Os resultados obtidos neste trabalho corroboram com achados de Volpato e Figueiredo (2005) e Cibulka et al. (2011), que relataram a importância de ações preventivas voltadas para a educação em saúde bucal, através de avaliação e higiene bucal durante a gravidez, auxiliando na melhoria da condição bucal das gestantes.

Em relação ao início do pré-natal por parte das gestantes primíparas, grande parte das entrevistadas deste estudo demonstrou interesse na busca de novas informações, realizando o acompanhamento a partir do primeiro trimestre de gravidez. A justificativa dada pelas próprias gestantes participantes baseou-se na novidade e no cuidado com o primeiro filho.
Houve grande interesse na realização do pré-natal no grupo de gestantes multíparas do estudo. Esse cuidado é relatado tanto em gestações anteriores quanto na atual, em que as mães aderiram e entenderam a importância da realização desse acompanhamento desde o início da gestação. Apenas um pequeno percentual de grávidas multíparas iniciou o pré-natal por volta do segundo e terceiro trimestre, relacionando este atraso em função da "experiência" de gestações passadas.

Em decorrência do rápido crescimento durante o primeiro ano de vida, a infância torna-se um dos períodos mais críticos do ciclo vital. Dessa forma, a nutrição e, mais especificamente, a alimentação natural são essenciais para o desenvolvimento da dentição e suas estruturas de suporte, bem como para o desenvolvimento humano. Segundo a OMS, para que o início e o estabelecimento do aleitamento tenham êxito, as mães necessitam do apoio ativo, durante a gravidez e após o parto, não apenas de suas famílias e comunidades, mas também de todo o sistema de saúde (CRUZ et al., 2010; NAGARAJ; PAREEK, 2012).

Na realização do pré-natal, as mães primíparas do estudo receberam orientações de como amamentar, sendo grande parte dessa orientação feita por profissionais da área médica. Todas as gestantes pretendem amamentar no peito, estipulando um período de até seis meses e realizar quatro vezes ou mais por dia, e escolhendo a forma de aleitamento misto. O aleitamento materno oferece ao bebê um adequado desenvolvimento ósseo e muscular, garantindo, assim, a inter-relação entre o sistema estomatognático e os demais órgãos e funções (MELO et al., 2002; BATISTA et al., 2011). Para o desenvolvimento do sistema estomatognático, a recomendação da Organização Mundial da Saúde é manter o aleitamento exclusivo até o sexto mês de vida. A falta da amamentação constitui-se num dos principais fatores etiológicos das más oclusões dentárias (ORGANIZAÇÃO MUNDIAL DA SAÚDE, 2017). 
As gestantes multíparas, embora tenham amamentado no peito até o sexto mês, também se utilizaram do aleitamento artificial, corroborando com os relatos de Cruz et al. (2010), apesar da conhecida importância da amamentação para a saúde da díade mãe-bebê, apontam para a existência de uma alta prevalência de desmame precoce. Uma das causas apontadas para o desmame precoce é a falta de conhecimento da mãe a respeito da qualidade do seu leite e da importância deste para o desenvolvimento sadio do bebê (BATISTA et al., 2011).

É evidente que o tratamento odontológico durante a gravidez é seguro e irá proporcionar uma melhor saúde bucal das mães. As gestantes primíparas mostraram-se preocupadas com a sua saúde bucal no período gestacional, além de possuir grande discernimento em relação ao tratamento odontológico na gravidez, uma vez que a maioria delas, neste estudo, relatou que o mesmo pode ser realizado durante a gestação, derrubando mitos e medos. Porém, mais da metade das entrevistadas não buscou atendimento, talvez porque já realizaram o pré-natal odontológico ou por fatores socioeconômicos, socioculturais, falta de discernimento em relação à importância da saúde bucal e preocupações com o bebê durante o tratamento odontológico (GEORGE et al., 2013).

As gestantes multíparas participantes desta pesquisa, quando indagadas sobre o tratamento odontológico durante a gestação, afirmaram majoritariamente acreditar que o atendimento pode ser feito sem intercorrências nesse período, mostrando conhecimento em relação ao importante papel desenvolvido pelo cirurgião-dentista no que tange à educação em saúde bucal. O pré-natal odontológico inclui ações, como desmistificar crenças e preocupações sobre a gravidez e o tratamento odontológico, conscientizar a respeito dos problemas bucais, orientar a importância do controle da placa e o uso do flúor, bem como cuidados com o futuro bebê (HASHIM, 2012).

Assim, as condições de saúde bucal e sistêmica, bem como informações físicas, sociais e psicológicas, podem sinergicamente melhorar a qualidade de vida relacionada à saúde de mães e filhos recém-nascidos. Destaca-se, para isso, a necessidade premente de estímulo e consolidação de uma promoção de saúde bucal efetiva, por meio de programas governamentais concentrados no público alvo deste estudo, as gestantes (AMRITA-GEEVARGHESE et al., 2017; MAZZONI; CARTER, 2017).

\section{Conclusão}

Constatou-se que as mulheres grávidas, tanto pela primeira vez como mães de mais filhos, realizam o pré-natal regularmente a partir do primeiro trimestre de gravidez e entendem a importância do atendimento odontológico.

\section{Referências}

AMERICAN ACADEMY OF PEDIATRIC DENTISTRY - AAPD. Definition, oral health policies and clinical guidelines, 2015.9p. Disponível em: <http://www.aapd.org $>$. Acesso em: 07 de nov. 2015.

AMRITA-GEEVARGHESE, A.; BASKARADOSS, J. K.; SARMA, P. S. Oral Health-Related Quality of Life and Periodontal Status of Pregnant Women. Maternal and Child Health Journal, 2017. http:// dx.doi.org/10.1007/s10995-016-2255-y.

BAMANIKAR, S.; KEE, L. K. Knowledge, attitude and practice of oral and dental healthcare in pregnant women. Oman Medical Journal, v. 28, n. 4, p. 288-291, 2013.

BASTIANI, C. et al. Conhecimento das gestantes sobre alterações bucais e tratamento odontológico durante a gravidez. Clin.Cient, v. 9, n. 2, p. 155160, 2010.

BATISTA, C. R. V.; TRICHES, T. C.; MOREIRA, E. A. M. Desenvolvimento bucal e aleitamento materno em crianças com fissura labiopalatal. Revista Paulista de Pediatria : Orgao Oficial da Sociedade de Pediatria de Sao Paulo, v. 29, n. 4, p. 674-679, 2011.

CATÃO, C. D. S. et al. Evaluation of the knowledge of pregnant women about the relationship between oral diseases and pregnancy complications. Revista de Odontologia da UNESP, v. 44, n. 1, p. 59-65, 2015.

CIBULKA, N. J. et al. Improving oral health in low-income pregnant women with a nurse practitioner-directed oral care program. Journal of the American Academy of Nurse Practitioners, v. 23, p. 249-257, 2011. 
CODATO, L. A. B. et al. Atenção odontológica à gestante: papel do profissional de saúde. Ciencia \& Saude Coletiva, v. 16, n. 4, p. 2297-2301, 2011.

CRUZ, S. H. et al. Orientação sobre amamentação: a vantagem do Programa de Saúde da Família em municípios gaúchos com mais de 100.000 habitantes no âmbito do PROESF. Revista Brasileira de Epidemiologia, v. 13, n. 2, p. 259-267, 2010.

FINKLER, M.; OLEINISKI, D. M. B.; RAMOS, F. R. S. Saúde Bucal Materno Infantil: Um estudo de representações sociais com gestantes. Texto \& Contexto Enfermagem, v. 13, n. 3, p. 360-368, 2004.

GEORGE, A. et al. The oral health status, practices and knowledge of pregnant women in southwestern Sydney. Australian Dental Journal, v. 58, p. 26-33, 2013.

HASHIM, R. Self-reported oral health, oral hygiene habits and dental service utilization among pregnant women in United Arab Emirates. International Journal of Dental Hygiene, v. 10, p. 142-146, 2012.

LANDERDAHL, M. C. et al. A percepção de mulheres sobre atenção pré-natal em uma Unidade Básica de Saúde. Escola Anna Nery, v. 11, n. 1, p. 105-111, 2007.

MAZZONI, S. E.; CARTER, E. B. Group Prenatal Care. American Journal of Obstetrics and Gynecology, 2017. http://dx.doi.org/10.1016/j. ajog.2017.02.006.

MEDEIROS, U. V.; ZEVALLOS, E. F. P.; ROSIANGELA, K. Promoção da saúde bucal da gestante: garantia de sucesso no futuro. Rev. Cien. do Cro-RJ, v. 2, p. 47-57, 2000.

MELO, A. M. C. A. et al. Conhecimentos e atitudes sobre o aleitamento em primíparas da cidade do Recife, Pernambuco. Revista Brasileira de Saú de Materno Infantil, v. 2, n. 2, p. 137-142, 2002.

MOIMAZ, S. A. S. et al. Relação entre aleitamento materno e hábitos de sucção não nutritivos. Ciencia \& Saude Coletiva, v. 16, n. 5, p. 2477-2484, 2011.

NAGARAJ, S.; PAREEK, S. Infant oral health knowledge and awareness: disparity among pregnant women and mothers visiting a Government Health Care Organization. International Journal of Clinical Pediatric Dentistry, v. 5, n. 3, p. 167-172, 2012.

ORGANIZAÇÃO MUNDIAL DA SAÚDE OMS. Recomendações da Organização Mundial da Saúde a respeito de aleitamento materno, 2010. Disponível em: <http://www.unicef.org/ programme/ breastfeeding/baby.htm $>$. Acesso em: 06 mar de 2017.

VERGNES, J. N. et al. Frequency and risk indicators of tooth decay among pregnant women in France: A cross-sectional analysis. PLoS One, v. 7, n. 2, p. 1-7, 2012.

VOLPATO, L. E. R.; FIGUEIREDO, A. F. Estudo da Clientela do programa de atendimento odontológico precoce em um serviço público do município de Cuiabá, Mato Grosso. Revista Brasileira de Saú de Materno Infantil, v. 5, n. 1, p. 45-52, 2005. 